ISSN No:-2456-2165

\title{
Traffic Flow Pattern in Road Network Using Clustering
}

\author{
${ }^{1}$ Ganga Gudi, \\ Department of Computer Science, \\ KLE’s S.Nijalingappa College, Bangalore, India.
}

\begin{abstract}
Wireless communication has become important in location-based services. The enormous amount of data is extracted for useful information to solve the real world problem. Global positioning system, is used to captures the position of an object at specific time period. The scheme is finding the congested route by considering the number of vehicles in a road segment. It consists of two methods, firstly it finds the group of points based on consistency of route points and second it arranges the groups in sequence of values for each route.
\end{abstract}

Keywords:- Trajectory points; Location Based Service (LBS); Traffic Pattern; pattern-mining.

\section{INTRODUCTION}

The moving object data is available with the help of GPS and mobile communication. One of the spatiotemporal data mining is to analyze data sets for detecting interesting patterns. Trajectory objects of moving objects can provide useful information for high quality location-based services (LBS). The work is divided in two methods firstly, where in it groups the points based on consistency of route and secondly, it arranges the groups in sequence of values for each route.

\section{PROBLEM DEFINITION}

Let $\mathrm{DT}=\mathrm{T} 1, \mathrm{~T} 2, \mathrm{~T} 3, \ldots, \mathrm{Tn}$ be a database of route, where in each $\mathrm{Ti}$ is a sequence of triples (xi, yi, ti) for $\mathrm{i}=1$, $2, .$, n. (xi, yi) gives a position of an object with instance of time ti . It groups the route points in same direction, in such a way that the route points inside each group must be density reachable from their respective group. Groups with the number of route points exceeding the minimum traffic threshold are removed.

\section{ALGORITHMIC FRAMEWORK}

\section{Algorithm: Disc-Cluster}

In this algorithm, DT=T1, T2,..,Tn be a database of route, where each $\mathrm{Ti}$ is a sequence of triplets (xi,yi,ti) for $\mathrm{i}=1,2, \ldots, \mathrm{n}$ such that (xi,yi) gives a position of a moving object at any instance of time ti. Group starts with $\mathrm{C} 0=$ $\{(\mathrm{x} 0 \mathrm{i}, \mathrm{y} 0 \mathrm{i}, \mathrm{t} 0 \mathrm{i})\}$ where $(\mathrm{x} 0 \mathrm{i}, \mathrm{y} 0 \mathrm{i}, \mathrm{t} 0 \mathrm{i})$ is the randomly selected trajectory $\mathrm{Ti}$ in a randomly selected path.
${ }^{2}$ Dr Hanumanthappa M,

Department of Computer Science and Applications, Jnana Bharti Campus Bangalore University, Bangalore, India.

Here, (xi,yi,ti) is compared with the other route points of the group and also with the points of same trajectories $\mathrm{Ti}$ except the point $(\mathrm{x} 0 \mathrm{i}, \mathrm{y} 0 \mathrm{i}, \mathrm{t} 0 \mathrm{i})$, such that $\operatorname{sqrt}\left(\mathrm{x}_{\mathrm{j}}-\mathrm{x} 0_{\mathrm{i}}\right)^{2}+\left(\mathrm{y}_{\mathrm{j}}\right.$ $\left.-\mathrm{y} 0_{\mathrm{i}}\right)^{2} \leq \sigma$

\section{$>$ Arranging Clusters:}

The Disc-Group algorithm provides all the possible groups in each route. These groups represent the regions where the traffic flow is very high.

\section{> Algorithm 1: Disc-Group $(D T, \sigma, \alpha)$ \\ - Group discovering algorithm.}

The route database DT $=\{\mathrm{T} 1, \mathrm{~T} 2, \mathrm{~T} 3, \ldots, \mathrm{Tn}\}$ where trajectory $\mathrm{Ti}=\{(\mathrm{xi} 1, \mathrm{yi} 1, \mathrm{ti} 1),(\mathrm{xi} 2, \mathrm{yi} 2, \mathrm{ti} 2), \ldots,(\mathrm{xim}, \mathrm{yim}$, tim) $\}$ and id and routeid are the id of the vehicle that generated route and its adjacent route.

Begin:

1: Arrange and sort $\mathrm{Ti}$ according to temporal values

$2: \mathrm{k} \leftarrow-1$

$3: \mathrm{s} \leftarrow 0$ \{ $\mathrm{s}$ is a group number $\}$

4: for all Ti $\varepsilon$ DT: $1 \leq \mathrm{i} \leq \mathrm{n}$ do

5: for points pt (xip, yip, tip) $\varepsilon \mathrm{Ti}: 1 \leq \mathrm{p} \leq \mathrm{m}$ do

6: if ptis not in not in any cluster formed then

7: $\mathrm{k} \leftarrow \mathrm{k}+1, \mathrm{~s} \leftarrow \mathrm{k}$ and add Pt to Cs.

8: else

9: $\mathrm{s} \leftarrow$ group number which contains $\mathrm{Pt}$

10: end if

11: for all $\mathrm{Tj} \varepsilon \mathrm{DT}: 1 \leq \mathrm{j} \leq \mathrm{n}$ do

12: if routeid and dom of $\mathrm{Ti}, \mathrm{Tj}$ are same then

13: for all points $\mathrm{pt} 1$ (xjq, yjq, tjq) $\varepsilon \mathrm{Tj}: 1 \leq \mathrm{q} \leq \mathrm{m}$ do

14: if $\operatorname{sqrt}(x j q-x i p)^{2}+(y j q-\text { yip })^{2} \leq \sigma$ and $\mid$ tjq - tip $\mid \leq \varepsilon$ then

15: Add point (xjq, yjq, tjq) to cluster Cs.

16: else

17: exit for

18: end if

19: end for

20: end if

23: end for

24: end for

\section{- Pattern-Mining}

Pattern-mining separates the group of each route and directions and mean of time values is calculated for each group. 
Algorithm 2: Pattern-Mining(C, routeids, dom) An Algorithm for mining traffic flow patterns.

Set of groups $\mathrm{C}=\{\mathrm{C} 1, \mathrm{C} 2, \ldots, \mathrm{Cn}\}$ obtained through algorithm Disc-Clusters, where $\mathrm{Ci}=\{(\mathrm{x} 1, \mathrm{y} 1, \mathrm{t} 1), . .,(\mathrm{xp}, \mathrm{yp}, \mathrm{tp})$, routeidj, domj \}.

\section{Begin:}

1: for all routeid, routeidj $\varepsilon$ routeids; $1 \leq \mathrm{j} \leq \mathrm{m}$ do

2: for all direction of movements, dmk $\varepsilon$ dom ; $1 \leq \mathrm{k} \leq 23$ :

for all clusters $\mathrm{Ci} \varepsilon \mathrm{C} ; 1 \leq \mathrm{i} \leq \mathrm{n}$ do

4: if routeidi $=$ routeidj and domi $=\mathrm{dmk}$ then

5: tmeani $\leftarrow \sum^{\mathrm{p}} \mathrm{l=1}$ tl

6: end if

7: end for

8: for all cluster $\mathrm{Ci} \& \mathrm{C}: 1 \leq \mathrm{i} \leq \mathrm{n}$ do

9: if routeidi $=$ routeidj and domi $=\mathrm{dmk}$ then

10: Arrange and sort groups according to tmeani

11: end if

14: end for

15: end for

16: end for

\section{CONCLUSION}

In this paper we presented a traffic flow pattern in a road network which will remove the heavy traffic regions using the group clustering algorithm and the arrange the groups in a particular sequence for different routes.

In this paper, as the algorithm identifies the heavy traffic regions in network, it groups the route points rather than the routes which give more information about the traffic area. This work can be planned with the route to each destination based on the traffic. This algorithm can also be explored with the large traffic real dataset.

\section{REFERENCES}

[1]. T. Brinkhoff, "A framework for generating networkbased moving objects, "GeoInformatica, vol. 6, no. 2, pp. 153-180, 2002.

[2]. G.-P. Roh, J.-W. Hwang and B.-K. Yi, "Supporting pattern-matching queries over trajectories on road networks, "IEEE Transactions on Knowledge and Data Engineering, vol, 23, no.11, pp. 1753-1758, 2011.

[3]. Cheng H, Yan X, Han J, Philip S (2008) Direct discriminative pattern mining. for effective classification.

[4]. Giannotti F, Nanni M, Pedreschi D - Trajectory pattern mining.

[5]. M Huang, P. Hu and L. Xia, "A grid based trajectory method for moving objects on fixed network", in Proceedings of the $18^{\text {th }}$ International Conference on GeoInformatics, June 2010.

[6]. Coppi R, D’Urso P - Fuzzy K-means clustering models for triangular fuzzy time trajectories. Stat Methods Appl 11(1), pp. 22-40. 